\title{
Efficient Time-Domain Modeling and Simulation of Passive Bandpass Systems
}

\author{
Yinghao Ye*†, Domenico Spina*, Dirk Deschrijver*, Wim Bogaerts ${ }^{\dagger}$, and Tom Dhaene* \\ *IDLab, Department of Information Technology, Ghent University - imec, Gent, Belgium. \\ ${ }^{\dagger}$ Photonics Research Group, Department of Information Technology, Ghent University - imec, Gent, Belgium.
}

Email: yinghao.ye@ugent.be

\begin{abstract}
In communication systems, the signals of interest are often amplitude and/or phase modulated ones. In this framework, the baseband equivalent signals and systems representation is usually adopted to simulate the digital parts of communication systems in an efficient manner. This contribution extends the applicability of such representation to RF/analog devices, leading to a common and efficient modeling and simulation framework. In particular, the proposed method can build half-size models compared to existing approaches, and allows one to choose the simulation time step according to the bandwidth of the modulating signals rather than the carrier frequency, thereby significantly speeding up the simulation procedure. The novel proposed method is validated via a suitable application example.
\end{abstract}

Index Terms-Baseband modeling and simulation, modulated signals, state-space representation, time-domain analysis.

\section{INTRODUCTION}

In communication systems, the signal of interest is often modulated on a carrier with frequency $f_{c}$, where the bandwidth of such modulating signal is often much smaller than $f_{c}$. Nowadays, telecommunication systems are moving to higher carrier frequencies for larger channel bandwidth. For example, the 5G network will operate at two frequency ranges: sub 6 $\mathrm{GHz}$ (with the maximum channel bandwidth of $100 \mathrm{MHz}$ ) and mmWave ( $>24 \mathrm{GHz}$, with a minimum channel bandwidth of $50 \mathrm{MHz}$ and a maximum of $400 \mathrm{MHz}$ ). For fiber-optic communication links, the optical carrier frequency is even in the range of $200 \mathrm{THz}$, with channel bandwidths of $100 \mathrm{GHz}$ or less. The time step for a direct simulation of these systems is mainly determined by the carrier frequency, which indicates that higher $f_{c}$ results in smaller time steps and larger computational complexity. In this scenario, baseband equivalent signals and systems are widely used in the simulation of communication systems to simplify the modulation, demodulation and filtering process [1]. The main idea is to "remove" the carrier frequency from the (bandpass) modulated signals and from the frequency response of the corresponding systems to efficiently process the information. This approach is efficient and accurate when dealing with the digital parts in a system, such as digital filters. However, it is not straightforward to apply the technique to the simulation of RF/analog parts (e.g. microwave filters in the front-end circuits) to achieve efficient time-domain simulations [1], [2].
A first approach to model passive photonic integrated circuits where electronic signals are modulated on optical carriers is proposed by us in [2], [3]. It starts from the scattering parameters of the photonic circuits under study, and derives a baseband equivalent model in state-space form which allows to conduct time-domain simulations in baseband regardless of the carrier frequency. Despite being developed for optical devices, this technique can be applied to linear and passive electronic systems as well, since it is based on a scattering parameters representation.

In this contribution, a novel alternative approach is presented, that is based on the Complex Vector Fitting (CVF) algorithm, and preserves all the advantages of the previous method [2], [3]. However, it generates more compact baseband state-space models, indicatively half the size of the ones obtained via [2], [3]. The paper is organized as follows. Section II describes the direct time-domain simulation of bandpass systems and the issue. The concept of baseband equivalent signals and systems is introduced in Section III. Section IV proposes the novel compact baseband modeling and simulation technique while an application example is provided in Section V. Conclusions are drawn in Section VI.

\section{BANDPASS SIGNALS AND SYSTEMS}

An amplitude and/or phase modulated signal with carrier frequency $f_{c}$ can be represented as

$$
a(t)=A(t) \cos \left(2 \pi f_{c} t+\phi(t)\right),
$$

where $A(t)$ and $\phi(t)$ are the time-varying amplitude and phase, respectively. Signals in the form (1) are referred to as bandpass signals [1], since the spectrum of $a(t)$ is centered around the carrier frequency and, typically, its bandwidth is relatively small compared to $f_{c}$.

Analogously, systems with input/output signals in the form (1) can be called bandpass systems. For passive bandpass microwave systems, the scattering parameters are widely used to represent their behaviors; hence, let us assume that the scattering parameters of the passive bandpass system under study have been obtained (via either simulations or measurements) at a discrete set of frequency values: $\boldsymbol{S}\left(f_{r}\right)$ for $r=1, \ldots, R$. 
Then, a pole-residue model can be built via the Vector Fitting (VF) technique [4]-[6]

$$
\boldsymbol{S}(s)=\sum_{k=1}^{K} \frac{\boldsymbol{R}_{k}^{V F}}{s-p_{k}^{V F}}+\boldsymbol{D}^{V F},
$$

where $s=j 2 \pi f$ is the Laplace variable, $p_{k}^{V F}$ are the (common) poles and $\boldsymbol{R}_{k}^{V F}$ are the corresponding residues, that are either real or complex conjugate pairs, while $D$ is a real matrix. Then, the VF model (2) can be easily converted into a time-domain state-space representation in the form

$$
\left\{\begin{aligned}
\frac{\mathrm{d} \boldsymbol{x}(t)}{\mathrm{d} t} & =\boldsymbol{A}^{V F} \boldsymbol{x}(t)+\boldsymbol{B}^{V F} \boldsymbol{a}(t) \\
\boldsymbol{b}(t) & =\boldsymbol{C}^{V F} \boldsymbol{x}(t)+\boldsymbol{D}^{V F} \boldsymbol{a}(t),
\end{aligned}\right.
$$

where $\boldsymbol{a}(t) \in \mathbb{R}^{n \times 1}$ and $\boldsymbol{b}(t) \in \mathbb{R}^{n \times 1}$ are the incident and reflected signals of the $n$-port bandpass system, respectively, while $\boldsymbol{x}(t) \in \mathbb{R}^{m \times 1}$ with $m=n K$ collects the state variables. The matrices $\boldsymbol{A}^{V F}, \boldsymbol{B}^{V F}, \boldsymbol{C}^{V F}, \boldsymbol{D}^{V F}$ can be analytically derived from (2) [7].

Time-domain simulations can be directly conducted by solving (3) via ordinary differential equation (ODE) solvers, where the maximum simulation time steps that can be adopted depends on the frequency of the input signals. Given that signals in the form (1) are considered in this contribution, the simulation time step depends on $f_{c}$ : if $f_{c}$ is very large, the simulation will be time consuming.

\section{BASEBAND EQUIVALENT SIGNALS AND SySTEMS}

To address the above challenge, a baseband modeling and simulation approach can be used to decrease the simulation cost by adopting relatively large time steps without losing accuracy [1]. The main idea is to "remove" the carrier frequency from the bandpass signal $a(t)$ by deriving a corresponding baseband equivalent signal $a_{l}(t)$ as

$$
a_{l}(t)=A(t) e^{j \phi(t)} .
$$

The relations between $a(t)$ and $a_{l}(t)$ are

$$
a(t)=\operatorname{Re}\left(a_{l}(t) e^{j 2 \pi f_{c} t}\right),
$$

where $\operatorname{Re}(\cdot)$ represents the real part. Note that $a_{l}(t)$ is the complex envelope of $a(t)$ [1]. The relation between $a(t)$ and $a_{l}(t)$ in the frequency domain is illustrated in Fig. 1.

The baseband equivalents of bandpass systems are defined in a similar way, as shown in Fig. 2, where $S(f)$ is the frequency response of the bandpass system represented by (2) and $S_{l}(f)$ is its baseband equivalent. If $a(t)$ is the port signal of the bandpass system $S(f), a_{l}(t)$ can be considered as the port signal of the baseband equivalent system $S_{l}(f)$ [1]. The main advantage of adopting baseband signals and systems for time-domain simulations is that the corresponding time-domain simulations can be carried out at baseband with relatively large time steps, and then the port signals of bandpass systems can be analytically recovered from the port signals of the baseband systems according to (5).

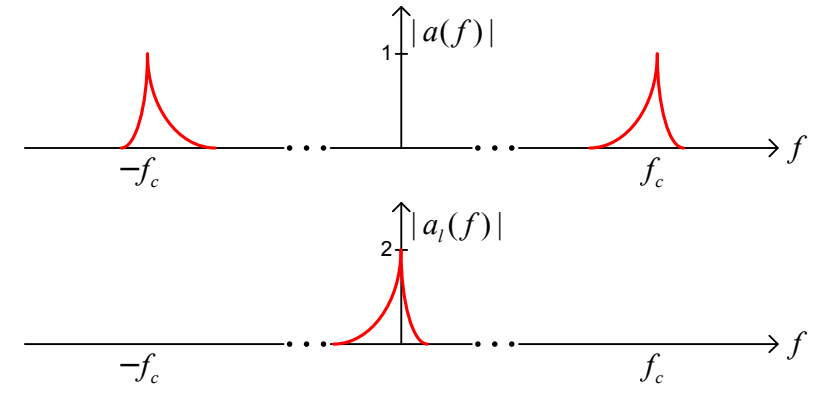

Fig. 1. Example of the amplitude spectrum of a bandpass modulated signal (top) and its baseband equivalent signal (bottom).

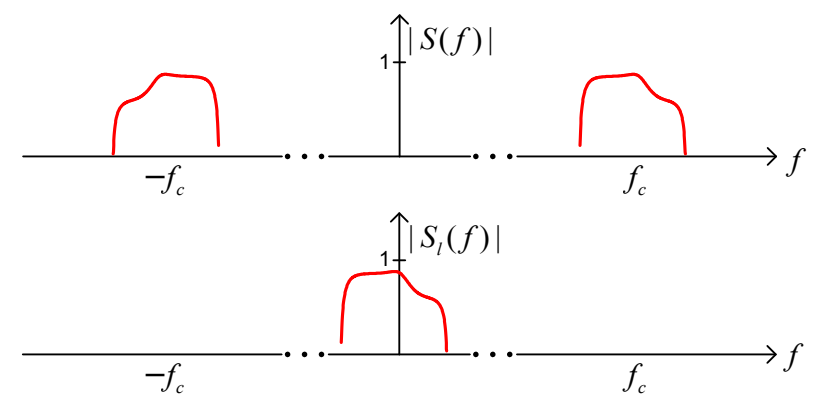

Fig. 2. Example of the amplitude spectrum of a bandpass system (top) and its baseband equivalent representation (bottom).

However, it is important to note that $S_{l}(f)$ has an asymmetric frequency response with regard to the positive and negative frequencies. As such, it represents a complex-valued system with a complex-valued impulse response. It is impossible to directly compute a model of $S_{l}(f)$ with the VF technique that models physical systems having a symmetric frequency response. In the next section, a novel approach to compute stable and passive baseband models of $S_{l}(f)$ is presented.

\section{Novel Modeling ApProach for BASEBAND EQUIVALENT SYSTEMS}

\section{A. Baseband Modeling Approach}

Consider the sampled bandpass scattering parameters $\boldsymbol{S}\left(f_{r}\right)$ for $r=1, \ldots, R$. The corresponding baseband representation $\boldsymbol{S}_{l}\left(f_{i}\right)$ can be obtained by shifting $\boldsymbol{S}\left(f_{r}\right)$ towards $0 \mathrm{~Hz}$ by an amount equal to the carrier frequency $f_{c}$ [1], as shown in Fig. 3 where $f_{i}=f_{r}-f_{c}$. Then, the CVF algorithm is developed to calculate a pole-residue model of $\boldsymbol{S}_{l}\left(f_{i}\right)$ as

$$
\boldsymbol{S}_{l}(s)=\sum_{k=1}^{K} \frac{\boldsymbol{R}_{k}^{C V F}}{s-p_{k}^{C V F}}+\boldsymbol{D}^{C V F},
$$

where $p_{k}^{C V F}$ are the (common) poles that can be either real or complex, and $\boldsymbol{R}_{k}^{C V F}$ are the corresponding residues, while $D^{C V F}$ is a real matrix. This form is very similar to the VF model (2), with one important difference: the poles and residues are not complex conjugate pairs. In this work, the CVF is a variant of the VF algorithm available at [8], which implements the techniques in [4]-[7], [9], [10]. Since VF has been extensively studied in the past two decades, and 


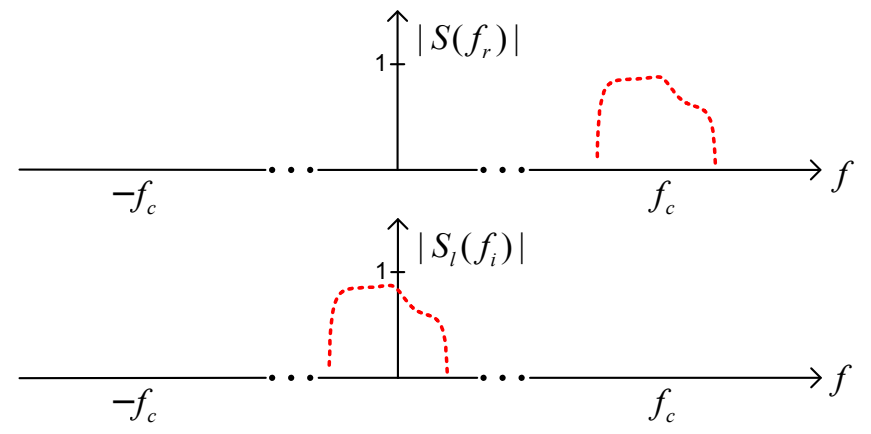

Fig. 3. The simulated or measured scattering parameters at a set of discrete frequency samples (top) and the corresponding baseband scattering parameters (bottom).

the proposed CVF shares several similarities with it, only the differences that are relevant for our application will be discussed in the following. Interested readers are referred to [4]-[7], [9], [10] for a thorough understanding of the VF modeling approach.

In particular, both CVF and VF adopt pole-residue models formed by real and complex poles having a negative real part, in order to guarantee the stability of the model [11]. For the VF technique, the complex poles and the corresponding residues must always occur in complex conjugate pairs to guarantee that the frequency response is always symmetric around zero (even amplitude and odd phase) and the corresponding impulse response in the time domain is real-valued: these are fundamental properties of linear and passive systems. However, baseband systems are non-physical and have an asymmetric frequency response with respect to $0 \mathrm{~Hz}$ by construction. Therefore, VF cannot be applied to modeling such systems directly [2], [3].

In order to overcome this problem, the complex conjugacy constraint on poles (and residues) is removed in the CVF algorithm. Besides this difference, the methodology employed to compute a pole-residue model is the same: the pole flipping scheme [4], relaxed formulation [9] and fast implementation base on QR decomposition [5] used in VF can be directly adopted for CVF.

The idea of removing the complex conjugacy constraint in the VF algorithm was first proposed in [12] in order to design complex infinite impulse response (IIR) filters having asymmetric frequency response. However, the models built in [12] are not used for time-domain simulations: the passivity definition, assessment and enforcement of the complex-valued models are not investigated in [12], while here they are rigorously studied in Section IV-B.

Once a model in the form (6) has been obtained via the CVF algorithm, it can be easily converted into a corresponding state-space form as

$$
\left\{\begin{aligned}
\frac{\mathrm{d} \boldsymbol{x}_{l}(t)}{\mathrm{d} t} & =\boldsymbol{A}^{C V F} \boldsymbol{x}_{l}(t)+\boldsymbol{B}^{C V F} \boldsymbol{a}_{l}(t) \\
\boldsymbol{b}_{l}(t) & =\boldsymbol{C}^{C V F} \boldsymbol{x}_{l}(t)+\boldsymbol{D}^{C V F} \boldsymbol{a}_{l}(t),
\end{aligned}\right.
$$

where $\boldsymbol{a}_{l}(t) \in \mathbb{C}^{n \times 1}$ and $\boldsymbol{b}_{l}(t) \in \mathbb{C}^{n \times 1}$ are the incident and reflected signals of the baseband system, respectively, which are the baseband equivalents of $\boldsymbol{a}(t)$ and $\boldsymbol{b}(t)$, while $\boldsymbol{x}_{l}(t) \in \mathbb{C}^{m \times 1}$ is the baseband equivalent of $\boldsymbol{x}(t)$. The matrices $\boldsymbol{A}^{C V F}, \boldsymbol{B}^{C V F}, \boldsymbol{C}^{C V F}, \boldsymbol{D}^{C V F}$ can be analytically obtained from (6), by using the same methodologies developed for the VF algorithm [7]. Now, time-domain simulations can be performed by solving (7) with relatively large time steps, since the spectrum of baseband signals and systems no longer depends on the carrier frequency. It is important to remark that the baseband model built by the proposed CVF technique is only half the size of the model built via the technique in [2], [3]. Indeed, the proposed technique only models the frequency response at positive frequency range while the technique in [2], [3] models the frequency response at both positive and negative frequency range as shown in Fig. 3.

\section{B. Passivity Assessment and Enforcement}

Since the baseband model (7) will be used for simulations in the time domain, the model must be passive [11]. In [2], [3], the passivity definition and conditions for complex-valued linear baseband systems are presented. In particular, there are two passivity constraints that baseband scattering parameters $\boldsymbol{S}_{l}(s)$ must satisfy:

1) $\boldsymbol{S}_{l}(s)$ is analytic in $\operatorname{Re}(s)>0$;

2) $\boldsymbol{I}_{n}-\boldsymbol{S}_{l}^{H}(s) \boldsymbol{S}_{l}(s)$ is a nonnegative-definite matrix for all $s$ such that $\operatorname{Re}(s)>0$.

where $\boldsymbol{I}_{n}$ is the identity matrix of size $n \times n$. Note that such conditions are the same as for physical systems, with the exception that the conjugacy relation $S^{*}(s)=\boldsymbol{S}\left(s^{*}\right)$ no longer needs to hold for complex-valued systems.

Now, the above passivity conditions require that the maximum singular value of $\boldsymbol{S}_{l}(s)$ is bounded by unity at all frequencies. In this framework, it has been proven that the Hamiltonian matrix $\boldsymbol{M}$ can be used to assess the model passivity with accuracy and efficiency, which is defined as

$$
\boldsymbol{M}=\left[\begin{array}{ll}
\boldsymbol{M}_{11} & \boldsymbol{M}_{12} \\
\boldsymbol{M}_{21} & \boldsymbol{M}_{22}
\end{array}\right]
$$

where

$$
\begin{aligned}
& \boldsymbol{M}_{11}=\boldsymbol{A}-\boldsymbol{B} \boldsymbol{L}^{-1} \boldsymbol{D}^{H} \boldsymbol{C}, \\
& \boldsymbol{M}_{12}=-\boldsymbol{B} \boldsymbol{L}^{-1} \boldsymbol{B}^{H}, \\
& \boldsymbol{M}_{21}=\boldsymbol{C}^{H} \boldsymbol{Q}^{-1} \boldsymbol{C}, \\
& \boldsymbol{M}_{22}=-\boldsymbol{A}^{H}+\boldsymbol{C}^{H} \boldsymbol{D} \boldsymbol{L}^{-1} \boldsymbol{B}^{H}, \\
& \boldsymbol{L}=\boldsymbol{D}^{H} \boldsymbol{D}-\boldsymbol{I}_{n}, \quad \boldsymbol{Q}=\boldsymbol{D} \boldsymbol{D}^{H}-\boldsymbol{I}_{n} .
\end{aligned}
$$

In particular, a (complex- or real-valued) stable state-space model is passive if its Hamiltonian matrix has no purely imaginary eigenvalues: any such eigenvalue indicates a crossover frequency where a singular value of the scattering matrix changes from being smaller to larger than unity, or vice versa [2], [7]. Once the crossover frequency points are identified by checking the eigenvalues of (8), the local maxima of violating singular values of the scattering matrix can be found [10]. Hence, passivity can be enforced by perturbing the residues such that the violating singular values become smaller than 


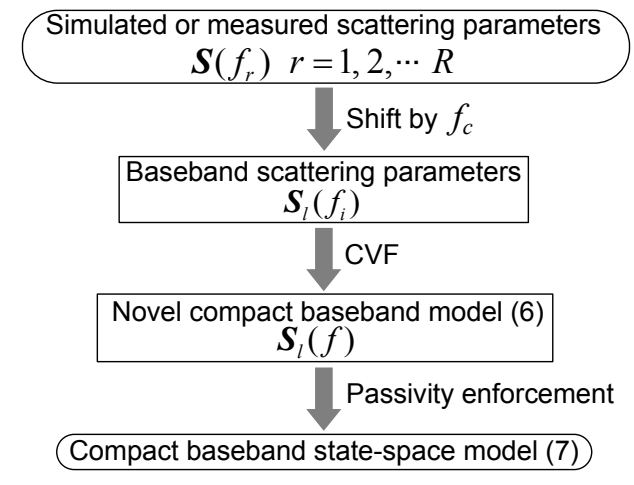

Fig. 4. The flowchart of the proposed modeling technique.

unity [10]. The flowchart of the proposed modeling strategy is shown in Fig. 4

\section{NumERICAL EXAMPLE}

Assume that the bandpass filter in Fig. 5 is used to filter incoming modulated signals and that its passband is centered at $12 \mathrm{GHz}$, with $3 \mathrm{~dB}$ bandwidth $1.27 \mathrm{GHz}$. First, the scattering parameters of the filter are evaluated in $\mathrm{ADS}^{1}$ in the frequency range $[9 ; 15] \mathrm{GHz}$. Following the proposed technique, the CVF baseband model in the form (7) is built with 6 poles, achieving a maximum absolute error of $-70 \mathrm{~dB}$ in magnitude. The accuracy of the built model is shown in Fig. 6.

For comparison purpose, a VF model is built with the same error threshold of $-70 \mathrm{~dB}$, leading to a 12 poles model in the form (3). Note that this VF model represents a bandpass system and is defined in the bandwidth $[9 ; 15] \mathrm{GHz}$, centered around the carrier frequency $f_{c}=12 \mathrm{GHz}$. Then, a baseband model (which will be indicated as "shifted" baseband model in the following) can be computed from the VF model according to [2], [3], which also has 12 poles.

Then, the filter is assumed to be excited at the left port by a voltage source with internal resistance $R_{s}=50 \Omega$, while the other port is terminated on a $R_{L}=50 \Omega$. The voltage source generates a modulated signal which is formed by modulating a pseudo-random sequence of 1000 bits (with a bit time of $4 \mathrm{ns)}$ on a carrier with $f_{c}=12 \mathrm{GHz}$. The time-domain simulations of the three models: the bandpass VF model (3), the shifted baseband model [2], [3], and the novel CVF baseband model (7), are performed in Matlab. Figure 7 illustrates the output voltages obtained from different models up to $60 \mathrm{~ns}$. Note that the port voltages and currents

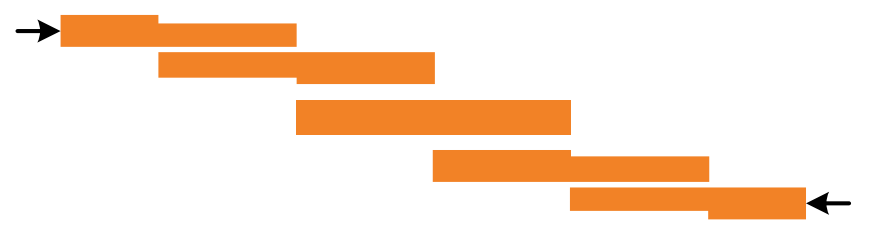

Fig. 5. The structure of the microstrip bandpass filter under study.

\footnotetext{
${ }^{1}$ Advanced Design System (ADS), Keysight Technologies.
}
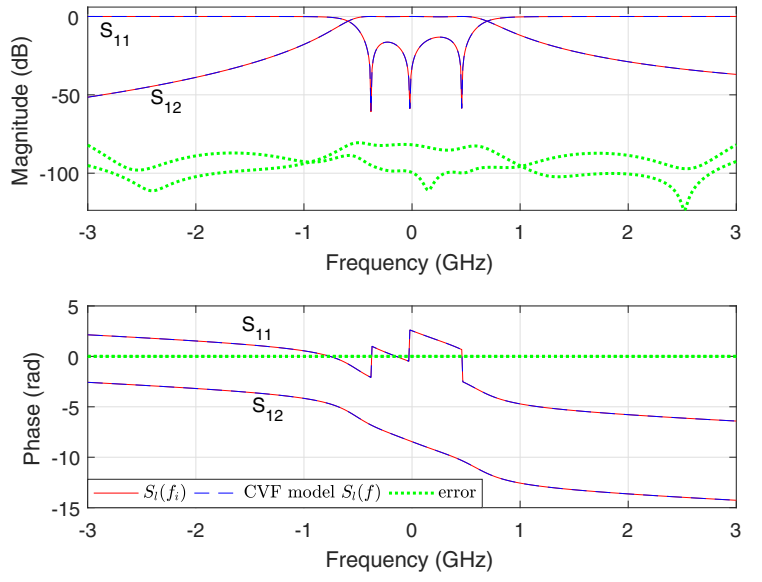

Fig. 6. The accuracy of the CVF baseband model $\boldsymbol{S}_{l}(f)$ with regard to baseband scattering parameters $\boldsymbol{S}_{l}\left(f_{i}\right)$.

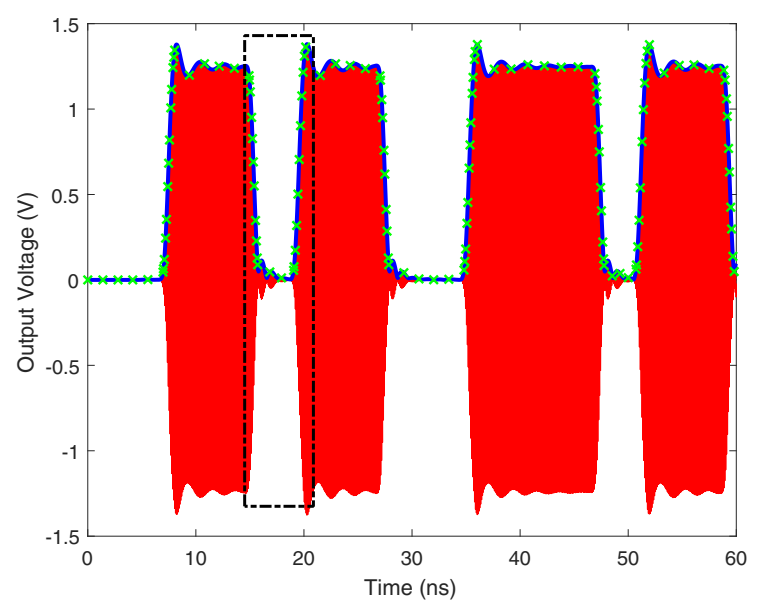

Fig. 7. The output voltage obtained from time-domain simulations of different models; the red line represents the output voltage from the bandpass VF model (3) which is a modulated signal, the blue line represents the amplitude of the output voltage from the novel CVF baseband model (7), while green crosses represent the amplitude of the output voltage from the shifted baseband model [2], [3].

from the shifted baseband model and the CVF model (7) are the baseband equivalents (and complex envelope [1]) of the port signals from the bandpass VF model (3), which is accurately demonstrated in Fig. 7. As mentioned in Section III, the modulated port signals of the filter can be analytically recovered from the port signals of the baseband models. This is demonstrated in Fig. 8, where the modulated output voltage is calculated from the baseband output and is compared to the modulated output voltage generated from the bandpass VF model (3), which shows an excellent agreement.

The time-domain simulation of the bandpass VF model (3) adopts a time step of $2 \mathrm{ps}$ and requires $2.7 \mathrm{~s}$, while the simulations of the shifted baseband model [2], [3] and the CVF baseband model (7) employ a time step of $40 \mathrm{ps}$ and require $0.4 \mathrm{~s}$ and $0.2 \mathrm{~s}$, respectively. Both baseband 


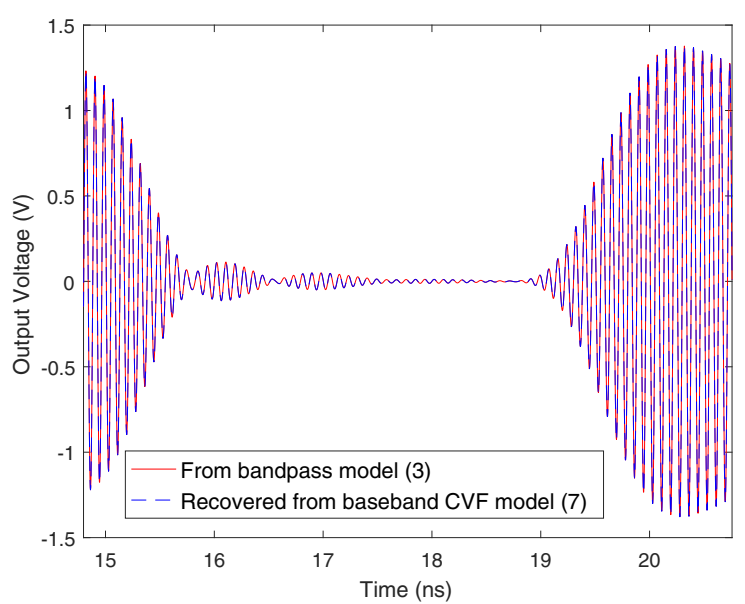

Fig. 8. The modulated output voltage of the filter, which is also a zoom of the area marked in Fig. 7.

models significantly outperform the bandpass model. When the number of poles of the two baseband models increases, the CVF model will outperform the shifted baseband model [2], [3] in terms of simulation efficiency, since the CVF model is only half the size of the shifted baseband model.

\section{CONCLUSION}

This paper extended the baseband modeling and simulation technique to RF/analog parts in communication systems. The CVF algorithm was proposed to build stable, passive and compact models of complex-valued baseband equivalents of analog bandpass systems. The advantage of the proposed technique is that the time steps for time-domain simulations of such systems can be chosen only according to the bandwidth of modulating signals rather than carriers. This allows to significantly reduce the simulation time, especially when the system has multiple ports and when the model requires many poles.

\section{REFERENCES}

[1] M. C. Jeruchim, P. Balaban, and K. S. Shanmugan, Simulation of communication systems: modeling, methodology and techniques. Springer, 2006.

[2] Y. Ye, D. Spina, Y. Xing, W. Bogaerts, and T. Dhaene, "Numerical modeling of a linear photonic system for accurate and efficient timedomain simulations," Photon. Res., vol. 6, no. 6, pp. 560-573, May 2018.

[3] Y. Ye, D. Spina, Y. Xing, W. Bogaerts, and T. Dhaene, "Fast and accurate time-domain simulation of passive photonic systems," in 2018 International Conference on Electromagnetics in Advanced Applications (ICEAA), Sep. 2018, pp. 396-399.

[4] B. Gustavsen and A. Semlyen, "Rational approximation of frequency domain responses by vector fitting," IEEE Trans. Power Del., vol. 14, no. 3, pp. 1052-1061, Jul. 1999.

[5] D. Deschrijver, M. Mrozowski, T. Dhaene, and D. De Zutter, "Macromodeling of multiport systems using a fast implementation of the vector fitting method," IEEE Microw. Compon. Lett., vol. 18, no. 6, pp. $383-$ 385, Jun. 2008.

[6] D. Deschrijver, B. Gustavsen, and T. Dhaene, "Advancements in iterative methods for rational approximation in the frequency domain," IEEE Trans. Power Del., vol. 22, no. 3, pp. 1633-1642, Jul. 2007.
[7] B. Gustavsen and A. Semlyen, "Fast passivity assessment for $S$ parameter rational models via a half-size test matrix," IEEE Trans. Microw. Theory Techn., vol. 56, no. 12, pp. 2701-2708, Dec. 2008.

[8] www.sintef.no/projectweb/vectfit/.

[9] B. Gustavsen, "Improving the pole relocating properties of vector fitting," IEEE Trans. Power Del., vol. 21, no. 3, pp. 1587-1592, Jul. 2006.

[10] — "Fast passivity enforcement for S-parameter models by perturbation of residue matrix eigenvalues," IEEE Trans. Adv. Packag., vol. 33, no. 1, pp. 257-265, Feb. 2010.

[11] S. Grivet-Talocia and B. Gustavsen, Passive Macromodeling: Theory and Applications. John Wiley and Sons, 2016.

[12] C.-U. Lei, C.-M. Cheung, H.-K. Kwan, and N. Wong, "Efficient complex continuous-time IIR filter design via generalized vector fitting," Proceedings of the International MultiConference of Engineers and Computer Scientists, vol. 2, 2008. 